\title{
Aproximación a la complejidad del alcohol y sus efectos en el comportamiento de los funcionarios policiales del municipio de Tulcán (Ecuador) ${ }^{*}$
}

\author{
Santiago Espinoza Borja** \\ Recibido: 08 de mayo del 2018 \\ Enviado a pares evaluadores: 15 de mayo del 2018 \\ Aprobado por Comité editorial: 30 de mayo del 2018 \\ https://doi.org/10.22395/csye.v7n13a2
}

\section{RESUMEN}

El presente artículo pretende abordar los efectos del alcohol en el comportamiento de los funcionarios encargados de hacer cumplir la ley en el distrito de Tulcán y así facilitar el proceso de retroalimentación institucional, imprescindible para los procesos evaluativos y de crecimiento. Se plantea la problemática como un fenómeno comportamental que no se origina exclusivamente en una sustancia, sino que resulta de múltiples vertientes que actúan de forma directa e indirecta en el desarrollo de la conducta consumista; en tal virtud, no se puede entender esto desde una visión lineal o tradicional, hace falta incorporar una teoría que conciba la complejidad. Para analizar estos efectos, se utilizan métodos y datos estadísticos cuyos resultados evidencian la tendencia decreciente de las faltas disciplinarias, y a la vez, los tipos de comportamientos relacionados con el alcohol que sanciona el Reglamento de Disciplina Institucional. Igualmente, se evidencia el efecto de bucle recursivo, mediante el cual la Institución actúa sobre sus miembros y a la vez, sus miembros influyen en la entidad policial, lo cual hace necesario acabar por completo con el consumo de alcohol dentro de las actividades convencionales y no convencionales que se realizan en la institución.

Palabras claves: alcohol; comportamiento; complejidad; funcionario; institución.

Este texto hace parte de una investigación realizada en el reparto policial del distrito de Tulcán, perteneciente a la provincia del Carchi, una de las 24 provincias del Ecuador, entre finales del 2016 y comienzos del 2017 en el contexto de la Maestría en Pensamiento Complejo de la Multiversidad Edgar Morin, México.

** Máster en Programación Neurolinguística de la Universidad Tecnológica Indoamérica (Ecuador). Especialista en Ciencias de la Complejidad de la Multiversidad Edgar Morin (México), donde se encuentra cursando maestría en Pensamiento Complejo. Licenciado en Psicología de la Universidad Particular de Loja (Ecuador). Hipnotista Eriksoniano con el aval del Instituto Milton Erickson de Argentina. Terapista EMDR con el aval del Instituto EMDR Iberoamérica. Correo electrónico: santi.borja@hotmail.es 


\section{An approach to the complexity of alcohol and its effects on the behavior of police officers in the municipality of Tulcán (Ecuador)}

\section{ABSTRACT}

This article addresses the effects of alcohol on the behavior of law enforcement officials in the Tulcán district to assist institutional feedback, which is essential for growth and evaluative processes. The problem is posed as a behavioral phenomenon that does not originate exclusively in a substance, but results from multiple variables that act directly and indirectly in the development of consumer behavior. In such virtue, this phenomenon may not be understood from a linear or traditional perspective, it is necessary to incorporate a theory that conceives complexity. To analyze these effects, statistical methods and data are used. Results show a decreasing tendency of disciplinary faults, and simultaneously evidence the types of behaviors related to alcohol that the Institutional Discipline Regulation sanctions. Likewise, the recursive loop effect, by means of which the Institution acts on its members and at the same time, its members influence the police entity they belong to, is made evident. Due to the above, completely stopping alcohol consumption within the conventional and non-conventional activities carried out in the institution becomes mandatory.

Keywords: alcohol; behavior; complexity; official; institution.

\section{Aproximação à complexidade do álcool e seus efeitos no comportamento dos funcionários policiais do município de Tulcán (Equador)}

\section{RESUMO}

O presente artigo pretende abordar os efeitos do álcool no comportamento dos funcionários encarregados de fazer com que se cumpra a lei no distrito de Tulcán e assim facilitar o processo de retroalimentação institucional, imprescindível para os processos avaliativos e de crescimento. Propõe-se a problemática como um fenômeno comportamental que não se origina exclusivamente em uma substância, mas que resulta de múltiplas vertentes que atuam de forma direta e indireta no desenvolvimento da conduta consumista. Em virtude disso, não se pode entendê-lo a partir de uma perspectiva linear ou tradicional, falta incorporar uma teoria que conceba a complexidade. Para analisar esses efeitos, foram utilizados métodos e dados estatísticos cujos resultados evidenciam a tendência decrescente das faltas disciplinares e, ao mesmo tempo, os tipos de comportamento relacionados com o álcool sancionados pelo Regulamento de Disciplina Institucional. Igualmente, fica evidente o efeito cíclico recursivo, por meio do qual a Instituição age sobre seus membros e, simultaneamente, seus membros influenciam na entidade policial, o que faz com que seja necessário acabar por completo com o consumo de álcool dentro das atividades convencionais e não convencionais realizadas na instituição.

Palavras-chave: álcool; comportamento; complexidade; funcionário; instituição. 


\section{Introducción}

Para efectos de este artículo se utiliza el término "conducta" como sinónimo de comportamiento, esto en función de la interpretación de la Real Academia Española de la Lengua (RAE, 2009), que define la conducta como "manera con que los hombres se comportan en su vida y acciones". La conducta se puede entender como la marca personal de cada individuo, es importante comprender que el comportamiento de cada sujeto se matiza por factores internos y externos como: creencias, valores, costumbres, experiencias, clima, lugar, horario, interacción personal, estado de salud, etc. Estos factores influyen a la persona a lo largo de su desarrollo individual y sientan la base de sus vivencias y de la forma particular de su actuar en los diferentes contextos de su diario vivir. Así, se puede decir que no se llega a conocer a una persona hasta conocer su conducta, tanto en sus momentos en soledad y de actuación en tanto individuo, como en aquellos que se desenvuelve en sociedad y en clave colectiva (comportamiento grupal). En esta línea, cuando Morin (2001) habla del "bien pensar" aclara:

Este es el modo de pensar que permite aprehender en conjunto el texto y el contexto, el ser y su entorno, lo local y lo global, lo multidimensional, en resumen, lo complejo, es decir las condiciones del comportamiento humano. (p. 59)

En este artículo se toma en cuenta un modelador de la conducta preponderante, como lo es la droga llamada alcohol.

$\mathrm{Al}$ abordar el consumo de esta droga y la alteración del comportamiento que suscita, se puede destacar que, gracias a las conductas visibles de las personas bajo los efectos de una droga, dicha alteración no es un tema desconocido. Pero, ¿qué se entiende por droga? Al respecto, la Organización Mundial de la Salud (OMS, 2004) tradicionalmente concibe que una droga es:

[...] toda sustancia que introducida en el organismo por cualquier vía de administración, produce una alteración, de algún modo, del natural funcionamiento del sistema nervioso central del individuo y es, además, susceptible de crear dependencia, ya sea psicológica, física o ambas.

Su clasificación no termina ahí, a lo largo de la historia las normas legales de cada país han ido aceptando como normal el consumo de un tipo de drogas y rechazando el de otras, esto ha llevado a que las drogas se clasifiquen de la siguiente manera: 1) drogas sujetas a fiscalización como marihuana, cocaína, hachís, etc. En países como Uruguay y Bolivia y en algunos Estados de Estados Unidos se acepta, con ciertas regulaciones, el consumo de marihuana o cocaína; y por otro lado, las drogas legales, como los cigarrillos y el alcohol.

Esta clasificación conlleva en cierta medida, que la gente conciba a las drogas permitidas como no drogas, los cual contribuyendo al aumento de su consumo. $\mathrm{El}$ alcohol, la droga más consumida en el mundo, es un caso particular de esto. 
En el Informe de situación regional sobre el alcohol y la salud en las Américas, la Organización Panamericana de la Salud y la Organización Mundial de la Salud (OPS y OMS, 2015) reportan que:

En la Región de las Américas, se consume más alcohol que en el resto del mundo. En particular, en los últimos cinco años han aumentado los episodios de consumo excesivo, pasando de una tasa del $4,6 \%$ al 13,0\% en el caso de las mujeres y del $17,9 \%$ al $29,4 \%$ en el de los varones. (p. 5)

Atendiendo a esta problemática, en países como España, específicamente en la ciudad de Madrid, De Puelles (2008) desarrolló un estudio dirigido a los cuerpos policiales titulado "Consumo perjudicial de alcohol en el Cuerpo Nacional de Policía: implicaciones disciplinarias, laborales y psicopericiales". De manera acorde, el presente artículo pretende abordar los efectos del alcohol en el comportamiento de los funcionarios encargados de hacer cumplir la ley en el distrito Tulcán, de esta manera contribuir en los procesos de retroalimentación policial y así coadyuvar a un mejor desarrollo de los métodos de evaluación, gestión y crecimiento institucional. Dicho propósito se lleva a cabo con una aproximación no tradicional (o de simplificación), con lo cual se evitan -al menos en parte-concepciones lineales de causa y efecto, puesto que se postula que esta realidad merece ser estudiada en toda su complejidad, entendiendo la complejidad desde la concepción del filósofo Edgar Morin:

La complejidad es la unión de la simplicidad y la complejidad, la unión de los procesos de simplificación (selección, jerarquización, separación, reducción) con sus contraprocesos (comunicación, articulación de lo que está disociado y es distinto). La complejidad va más allá de la alternativa entre el pensamiento reductor, que sólo [sic] ve los elementos, y el pensamiento global, que sólo [sic] ve el todo. (Munne, 2003, p. 8)

Es importante entender que la ocurrencia de un hecho se da gracias a un proceso sistemático de eventos, circunstancias y antecedentes previos, durante y posteriores al hecho.

Desde una visión patológica, la Organización Mundial de la Salud (OMS, 1992), mediante la Clasificación Internacional de Trastornos Mentales (CIE-10, 1992), establece la existencia de los Trastornos mentales y del comportamiento debidos al consumo de alcohol, en tal virtud, se ha de entender que al presentarse el consumo de alcohol en las filas policiales, el comportamiento de sus miembros puede verse afectado. En tal virtud, se procedió a recabar la información concerniente al número de sanciones disciplinarias de los años 2014, 2015 y 2016. Se seleccionaron las sanciones directamente relacionadas con el consumo de licor o estado de embriaguez ${ }^{1}$ y el ausentismo laboral (subsiste), lo

El eje lo constituyeron: los que se presentaren en público ante el personal formado o concentrado en estado de embriaguez, no asistir oportunamente a un servicio sin causa justificada o concurrir en estado de embriaguez, haber ingerido bebidas alcohólicas antes del servicio, etc. 
último debido a que solo se sancionan las inasistencias que no se pueden justificar adecuadamente.

\section{De la disciplina policial}

Para normar el comportamiento adecuado y el inadecuado, en todas las sociedades alrededor del mundo se han creado leyes, códigos, constituciones, acuerdos, entre otras normas que, en su mayoría, se orientan al castigo, advertencia, temor o reprensión, muy pocas de ellas a la recompensa del buen comportamiento. Dentro de las instituciones del orden público pasa de igual manera y la Policía Nacional del Ecuador no es la excepción; a través del Reglamento de disciplina de la Policía Nacional, se busca regir disciplinariamente la prevención de hechos negativos perpetrados por sus funcionarios. Este tipo de controles funcionan a manera de medida ejemplarizadora (promesa de castigo o sanción en caso de cometer una falta).

En relación con el Reglamento de disciplina como promesa de castigo para impedir comportamientos no adecuados de un servidor policial, en el 2014 se instituyeron nuevas disposiciones legales en materia penal en el país, tal como la aprobación que hizo la Asamblea Nacional del Ecuador (2014) del Código Orgánico Integral Penal, el cual establece "la prohibición de cualquier forma de privación de libertad en instalaciones o lugares no autorizados legalmente, así como toda forma de arresto, coerción o privación de libertad derivada de procedimientos disciplinarios administrativos" (Código Integral Penal, [COIP], 2014). De acuerdo con dicha prohibición, en la institución policial se elimina la figura de arresto como medida sancionadora y se creando una nueva clasificación de sanciones con respecto a las faltas de primera y segunda clase.

No obstante, esta reforma solo aplicó durante un breve periodo (dos años) por cuanto el 22 de diciembre del 2016, mediante el "Acuerdo Ministerial No 8010", entró en ejecución el nuevo Reglamento de Disciplina de la Policía Nacional, el cual acoge las modificaciones realizadas al reglamento de 1998 y a la vez, se fundamenta en el mismo, sin dejar de reconocer que se incorporan novedades en el discernimiento y sanción de las faltas disciplinarias. De esta manera, en el capítulo primero, titulo cuarto de este (2016), las sanciones de las faltas disciplinarias quedan clasificadas de la siguiente forma:

Artículo 31. Las faltas se reprimirán con las siguientes sanciones:

1) Destitución o baja;

2) Reprensión simple, formal o severa en sus diferentes niveles;

3) Suspensión del cargo sin goce de remuneración hasta 30 días;

4) Sanción pecuniaria del 1 al 7\% de la remuneración mensual unificada (Ministerio del Interior del Ecuador, 2016, p. 12). 
Con respecto al alcohol, en el presente Reglamento de Disciplina, el Artículo 30 recoge las agravantes de cometer un acto de indisciplina, su literal a) menciona como una de las agravantes: "cometer la falta en actos de servicio y en estado de embriaguez siempre que este no constituya delito".

Una vez establecidos los tipos de sanciones y agravantes en relación con el consumo de licor, se pueden mencionar algunas de las acciones que giran en torno al alcohol y que son consideradas una violación al Reglamento de Disciplina de la Policía Nacional (Ministerio del Interior del Ecuador, 2016), esto de acuerdo con la clasificación de las sanciones:

[...] se castiga el hecho de que un servidor policial haya ingerido bebidas alcohólicas antes del servicio, se presentare en público ante el personal formado o concentrado en estado de embriaguez, concurrir al trabajo en estado de embriaguez, consumir alcohol durante el servicio (trabajo), conducir vehículos oficiales en estado de embriaguez; entendiendo que todas estas sanciones son administrativas y no exceptúan al infractor de atenerse a las instancias legales. (Ministerio del Interior del Ecuador, 2016, pp. 21-26)

Es importante destacar el Artículo 8 de las Dispersiones Finales de este reglamento, el cual señala que "Es deber del superior preservar la comisión de infracciones y sólo [sic] como último recurso acudir a las sanciones. Así mismo, estimular a quien sobresalga en el cumplimiento de sus obligaciones" (Ministerio del Interior del Ecuador, 2016, p. 8).

\section{Sobre el alcohol: una droga no-droga}

En el transcurso del desarrollo de una persona, uno de los comportamientos que la sociedad le propone y transmite es la conducta consumista de alcohol, esto principalmente porque las bebidas embriagantes son permitidas por las leyes, se venden en un sinfín de lugares, son de fácil acceso, se consumen en toda ocasión y se ofrece una amplia variedad de razones para ello. Todo ello puede ofrecer una explicación al por qué los funcionarios encargados de hacer cumplir la ley consumen alcohol, después de todo Morin (1994) refirió que "[...] los individuos producen la sociedad, la que produce a los individuos" (p. 3). De igual manera, se puede argumentar que el hecho de ser policía no le quita la condición de persona, entendiendo el concepto persona desde la uni-multiplicidad de ser padre/madre, hijo, esposo/esposa, policía, individuo, amigo, hermano, familiar, grupo, etc.; pero también se podría llegar a señalar que es probable que el problema no sea el alcohol sino el uso o abuso del mismo.

El alcohol, una droga no-droga es un criterio que surge de la clasificación común que las personas realizan de las drogas, puesto que, a pesar de que desde la perspectiva y entendimiento del ámbito sanitario (salud, medicina) se 
habla de drogas (incluido el alcohol), cuando las personas en general se refieren a las drogas, la gran mayoría las relacionan con la marihuana, la cocaína o sus derivados. Dicha situación se evidencia también en la distinción que hacen organizaciones tradicionales como Alcohólicos Anónimos y Narcóticos Anónimos, los cuales surgen de manera consciente o inconsciente en función de esta clasificación que pareciera separar el alcohol de las otras drogas. Tanto así que al que consume licor se le denomina: "alcohólico", "borracho", "chumado", "tomador", "bebedor", entre otros. En cambio, a quien consume marihuana, cocaína o sus derivados se le denomina "drogadicto". Incluso en las campañas de prevención existe una marcada diferencia publicitaria para las drogas y para el alcohol, como si fuesen dos cosas distintas.

Si se parte del concepto de la OMS (s.f), droga es,

[...] cualquier sustancia que una vez introducida en el organismo, a través de distintas vías (inyectada, fumada o tragada), tiene capacidad para alterar o modificar las funciones corporales, las sensaciones, el estado de ánimo, o las percepciones sensoriales (vista, oído, tacto, gusto, olfato).

Así, el alcohol o alcohol etílico (etanol) mezclado en diferentes concentraciones en las bebidas como la cerveza, el vino y los licores destilados (aguardiente, brandy, ginebra, ron, tequila, vodka, whisky, etc.), es una droga depresora, y si bien sus efectos inmediatos son la euforia y la desinhibición, con la continuación de su consumo se genera una disminución del ritmo cardiaco y respiratorio, el tiempo de reacción es retardado, se desencadena la descoordinación y sedación del sujeto consumidor.

Se debe tener presente que el alcohol sigue siendo la droga de mayor consumo en el mundo (según estadísticas de la OMS) y a la vez, la droga que más daño social causa. Precisamente en el Ecuador, según el Ministerio de Salud Pública (MSP, 2016), en el 2010 el Consejo Nacional de Sustancias Estupefacientes y Psicotrópicas (Consep, 2010) informó:

En el año 2010, se reportaron 515 muertes atribuidas al consumo de sustancias psicotrópicas, diagnosticados y clasificadas por la Clasificación Internacional de Enfermedades (CIE-10), lo que viene a representar el 0,89\% del total de muertes. Dentro de este porcentaje el alcohol es el principal causante, atribuyéndole un 97,43\% de las causas. (MSP, 2016, p. 6)

Es importante entender que el alcohol, al tener característica soluble, pasa prontamente al sistema digestivo y posteriormente al sistema circulatorio. Al recorrer el cuerpo, sus efectos son inmediatos, es así que se puede evidenciar que en bajas cantidades, produce un engañoso efecto de seguridad, euforia y desinhibición, todas ellas sensaciones pasajeras a medida que se aumenta el consumo, ya que el alcohol es en esencia un depresor del sistema nervioso central 
(SNC). Lo anterior conlleva que en grandes ingestas de esta sustancia el SNC disminuye su control sobre el organismo, lo cual desemboca en que la persona tenga poca coordinación, una disminución de sus reflejos, dificultades de comunicación, fatiga, somnolencia, intoxicación, pérdida de conciencia e incluso puede llegar a la muerte por depresión cardiorrespiratoria; también se asocian otros efectos por el consumo crónico o recurrente como: impotencia, cirrosis, atrofia y degeneración cerebral. En las embarazadas puede derivar en síndrome alcohólico fetal, bajo nivel intelectual, malformaciones, etc. Es importante destacar que los efectos dependen de la cantidad, tipo y velocidad de la ingesta, así como del peso, sexo y forma de alimentación de la persona en cuestión.

Como afectación comportamental, es posible mencionar que el alcohol afecta los sentimientos, la memoria, procesos reflexivos, control social, y puede derivar en irritabilidad, impulsos sexuales, impulsos agresivos, inestabilidad emocional, deterioro de la capacidad de juicio, deterioro familiar y social, conductas delictivas, ausentismos laborales y suicidios.

\section{Visión del método}

El presente estudio tiene un enfoque metodológico cuantitativo-cualitativo, la información se recabó de la oficina de Recursos Humanos de la Sub Zona de Policía Carchi, Distrito Tulcán, que tuviesen relación con el listado de las sanciones impuestas a los servidores policiales durante los años 2014, 2015 y 2016; Luego se procedió a clasificar los datos obtenidos, extrayendo aquellos datos de las sanciones registradas en el Distrito Tulcán que presentaran relación directa con el consumo de alcohol o estado de embriaguez. De igual manera, se llevaron a cabo entrevistas a los funcionarios de asesoría jurídica y oficina de recursos humanos para determinar qué otras faltas disciplinarias tenían relación directa o indirecta con el consumo de alcohol.

De esta forma, gracias a la recolección de información cuantitativa (estadística de castigados) y también de la información cualitativa de las entrevistas que buscaban la identificación de comportamientos que se consideran contrarios a la reglamentación que rige la institución policial, fue posible conocer datos reveladores de una realidad institucional que como en el mundo entero, puede llegar afectar a la persona o servidor policial en su individualidad, al grupo de familia y a la sociedad al este pertenecer a un organismo gubernamental que tiene como misión hacer cumplir la ley.

\section{Resultados}

La Sub Zona de Policía Carchi (Provincia del Carchi) tiene en la actualidad un número aceptable de efectivos policiales, aun teniendo en cuenta la permanente 
e indistinta movilización de efectivos que son asignados a otras plazas, mientras otros agentes son designados a trabajar en la Sub Zona Carchi; se puede hacer una cuenta aproximada de entre 700 y 800 servidores policiales en esta sub zona. Este total se reparte en los tres Distritos: Espejo, Montufar y Tulcán, luego en circuitos y sub circuitos. A la vez, se puede hallar otra división con base en los servicios que prestan como: Policía Comunitaria, Antinarcóticos, Administración, Sanidad, Policía Judicial, Criminalística, Grupo Motorizado, entre otros.

Esta división no obedece a principios de separación, sino que más bien a un fundamento transdisciplinario. Al respecto, Max-Neef (2004, p. 7) explica que "[l]a transdisciplinaridad se da cuando existe una coordinación entre todos los niveles". Así ocurre en la policía porque el trabajo se realiza en coordinación mutua a fin de alcanzar objetivos que trasciendan aquellas necesidades individuales o sectoriales, y que porten a un solo objetivo general, el de cumplir con la misión de la institución, es decir de la Policía Nacional entendida como un todo. El Distrito Tulcán alberga el mayor número de efectivos policiales (entre 500 y 600), Montufar (entre 100 y 150) y Espejo (entre 50 y 60), esto contando los diferentes servicios policiales.

El análisis de estadísticas de los sancionados en los años 2014, 2015 y 2016 arrojó resultados interesantes que se presentan a continuación según su año respectivo para una mayor comprensión.

En el 2014 se registró un total de 235 sanciones en toda la Sub Zona Carchi, de estas 153 sanciones se dieron en el Distrito Tulcán, lo cual equivale al 65,10\%; de las 153 sanciones, 63 se relacionan con faltas originadas por el consumo de alcohol, es decir el 41,14\%. Cabe aclarar que bajo dicha categoría están: presentarse con aliento a licor, en estado de embriaguez y subsistes (ausencia por varios días al trabajo sin justificación, lo cual desde la experiencia de la Asesoría Jurídica, está relacionado en un $80 \%$ a $90 \%$ con el consumo de licor).

En el 2015, en la Sub Zona Carchi se registraron 115 sanciones por diferentes causas, de las cuales 90 sanciones fueron impuestas en el Distrito Tulcán, lo que equivale al 78,26\%, de estas faltas; entre ellas 36 atañen al consumo de alcohol, lo que equivale al $40 \%$.

En el 2016 hubo 105 sanciones disciplinarias en la Sub Zona Carchi, 94 impuestas en el Distrito Tulcán, lo cual equivale al 89,52\%, de estás, 28 sanciones tienen que ver con el consumo de alcohol, un porcentaje del $29,78 \%$ de las reprensiones en las cuales el licor pudo haber generado una alteración comportamental. 
Tabla 1. Estadística de sanciones de la SZ Carchi y Distrito Tulcán en los años 2014, 2015 y 2016 y las sanciones a causa del alcohol con su porcentaje respectivo

\begin{tabular}{lccc}
\hline \multicolumn{1}{c}{ Población } & Sanciones 2014 & Sanciones 2015 & Sanciones 2016 \\
\hline Sub Zona Carchi & $235(100 \%)$ & $115(100 \%)$ & $105(100 \%)$ \\
Distrito Tulcán & $153(65,10 \%)$ & $90(78,26 \%)$ & $94(89,52 \%)$ \\
Faltas referentes al alcohol & $63(41,14 \%)$ & $36(40 \%)$ & $28(29,78 \%)$ \\
\hline
\end{tabular}

Fuente: datos de la oficina de Recursos Humanos de la Sub Zona de Policía Carchi

\section{Análisis}

Al examinar estos resultados, el debate gira en torno a si el comportamiento inadecuado que originó estas faltas disciplinarias, estuvo exclusivamente afectado por la variable del alcohol o si se presentan otros factores como: el que los sujetos tuviesen dificultades o problemas (personales o laborales) previos al consumo de licor, que el exceso de confianza o la esperanza de no entrar en evidencia llevase al cometimiento de estas faltas. Las circunstancias socioambientales generan una multi-influencia que restringe la individualidad de las personas, ¿en verdad existe tal individualidad o solo somos un todo que responde al todo?, en fin, hay múltiples preguntas que formular al momento de analizar estos datos. Pero desde ya se puede establecer que esta conjugación de posibles razones escapa al método lineal causa-efecto, en tal virtud, no se puede caer en la simplificación que favorezca una de estas posibilidades para determinar que una u otra es la causal especifica que genera un comportamiento que se tilda de inadecuado.

Es necesario tener un panorama amplio para analizar los resultados, que si bien se fundamentan en los individuos, no por ello se debe dejar de analizar el todo. Al respecto, cabe resaltar el señalamiento de Pascal (s.f) citado por Edgar Morin (1999):

Como todo es causado y causante, ayudado y ayudante, mediato e inmediato y como todo se mantiene por un vínculo natural e insensible que relaciona a los más alejados y a los más diferentes, considero imposible conocer las partes sin conocer el todo y conocer el todo sin conocer particularmente las partes. (p. 98)

De acuerdo con lo anterior, más que solo ver las faltas disciplinarias de los integrantes de la Institución Policial, también se debe analizar la dinámica de la institución en su conjunto, al respecto, la Constitución del Ecuador (2008) establece en su Art. 63 que:

La Policía Nacional es una institución estatal de carácter civil, armada, técnica, jerarquizada, disciplinada, profesional y altamente especializada, cuya misión es atender la seguridad ciudadana y el orden público, y proteger el libre ejercicio de los derechos y la seguridad de las personas dentro del territorio nacional. (República del Ecuador, 2008) 
Se puntualiza entonces en la estructura jerarquizada y disciplinada que implica la obediencia al superior jerárquico, aquel que tiene la obligación de sancionar a un compañero, aún a sabiendas del malestar que esto puede causar en el sancionado, lo anterior puede generar un sesgo subjetivo por el cual se decida no sancionar a un subalterno, lo cual lleva a considerar que los datos expuestos en la tabla 1 (Estadística de sanciones en 2014, 1025 y 1026) pueden estar matizados por referir solo las faltas sancionadas, puede que se hayan dado otras acciones indebidas que no se sancionaron. Como se señaló anteriormente, el estudio tomó en cuenta solo las sanciones registradas y relacionadas directamente con el alcohol y los subsistes, entendiendo el alcohol como causal indirecta de los últimos.

Es así que según las estadísticas de la oficina de Recursos Humanos, se puede evidenciar un decremento anual en faltas disciplinarias en la Sub Zona Carchi en estos tres años, lo cual se contrapone con el incremento en el porcentaje de sanciones impuestas en el Distrito Tulcán; esto con la salvedad que en cambio, se presenta una disminución en las sanciones relacionas con el consumo de alcohol (consúltese tabla 1). Si bien las estadísticas obedecen a una percepción lineal en base a lo deseado -es decir, al querer bajar algo se espera que los resultados sigan la línea recta de la reducción, pero si el deseo es que haya un incremento, se espera que los resultados lineales sean de aumento y que puedan trascender hacia la conducta de las personas-, se diría que se espera un orden lógico sin tomar en cuenta que la naturaleza humana no podría ser lineal y ordenada.

Hemos aprendido a finales del siglo XX que hay que substituir la visión de un universo que obedece a un orden impecable por una visión donde el universo sea el juego y lo que está en juego es una dialógica (relación-antagónica, competente y complementaria) entre el orden, el desorden y la organización. (Morin, 1999, p. 21)

En función de lo expuesto no podríamos cantar victoria ni fracaso ante los resultados, tan solo entender que las estadísticas sobre el comportamiento humano no se pueden analizar linealmente, sino que se debe tomar en cuenta la dinámica en la cual se genera o desenvuelve la conducta bajo estudio.

Lo anterior conduce a hacer referencia sobre el entorno desde la Teoría General de Sistemas que al describir el ambiente, "[s]e refiere al área de sucesos y condiciones que influyen sobre el comportamiento de un sistema" (Arnold, y Osorio, 1998, p. 4). Así las cosas, cada persona es un sistema constituido por sistemas y a la vez es parte de sistemas más amplios (ambiente exterior). Desde esta perspectiva, se podría inferir la influencia que tiene el medio ambiente en los individuos, el cual puede incitar a los policías a consumir licor en un momento inoportuno, como por ejemplo horas antes de iniciar jornada laboral. Adicional a la presión externa, influye el hecho de que no exista un control permanente, lo cual podría impulsar al servidor policial a arriesgarse con la esperanza de 
no ser descubierto o de no recibir a una sanción. Esto conduce a los factores internos, es decir, la modalidad de consumo de cada persona que, a pesar de que el individuo la conozca, puede llegar a hacer una auto-negación para poder realizar un consumo determinado. Es decir, puede que la persona sepa que una vez acepta una copa de licor va sentir la necesidad de seguir consumiendo, pero para aceptar la primera copa de licor, se auto-engaña al creer que esta vez en serio, solo va tomarse esa copa.

Si se entiende que el alcohol causa efectos en los sentimientos, la memoria, procesos reflexivos, y control social; genera irritabilidad, impulsos sexuales, impulsos agresivos, inestabilidad emocional, deterioro de la capacidad de juicio, deterioro familiar y social, conductas delictivas, ausentismos laborales y suicidios; es de suponer que si los funcionarios encargados de hacer cumplir la ley consumen licor, van a tener afectaciones comportamentales que les lleven a incurrir en faltas disciplinarias. Esto va más allá de la causa-efecto, y depende en gran medida del momento de ingesta, cantidad de ingesta, horario de trabajo, reacciones individuales con el alcohol y otras circunstancias que puedan existir. Se suma a lo anterior que para que haya una sanción, debe haber un superior que esté convencido de que no existe justificación alguna para el hecho evidenciado. Así las cosas, una sanción posiblemente siempre será subjetiva en base a las agravantes y atenuantes consideradas por quien detenta el poder para imponer la sanción.

Se podría llegar a entender el bucle recursivo existente entre la institución como un todo y sus funcionarios como sus partes, el todo influiría en las partes motivando el consumo de licor desde procesos de interacción, invitaciones, ceremonias, reuniones y demás actividades en las cuales el alcohol sigue siendo uno de los protagonistas; pero al mismo tiempo, el todo sanciona el consumo de drogas y promueve campañas de prevención desde distintos estamentos como el Servicio de Sanidad; se generaría entonces un proceso de interdependencia entre consumo y control del consumo. Al respecto, Morin (2011) habla de una "auto-eco-organización" haciendo mención a la relación existente entre autonomía y dependencia.

\section{Conclusiones}

Mediante la revisión de la tabla 1, se puede evidenciar que existe un decremento de las sanciones disciplinarias impuestas a los servidores policiales, en la Sub Zona Carchi se ha bajado de 235 en el 2014 a 105 en el 2016. De igual manera, en el Distrito Tulcán se redujo de 153 (2014) a 94 (2016). Asimismo, existe una considerable reducción en el porcentaje de faltas disciplinarias cometidas en el Distrito Tulcán a causa del alcohol, es así que de 63 faltas sancionadas en el 2014 se pasa a 36 en el 2015 y se llega a 28 en el 2016.

- 62 
$\mathrm{El}$ alcohol es causante de una gran parte de las sanciones disciplinarias impuestas a los funcionarios encargados de hacer cumplir la ley en los periodos estudiados, en el 2014, el 41,14\% fueron a causa del alcohol, seguidamente en el 2015 el $40 \%$ y en el 2016 el 29,78\% de las faltas estuvo directamente relacionado al consumo de licor. Las sanciones tienen un alto nivel de subjetividad, esto porque están supeditadas a lo que el superior considere como una agravante o atenuante en una determinada falta.

El alcohol afecta el comportamiento de los servidores policiales, propicia de esta manera que ellos incurran en faltas disciplinarias como: no asistir oportunamente a un servicio sin causa justificada, concurrir en estado de embriaguez, presentarse en público, ante el personal formado o concentrado en estado de embriaguez, no asistir a trabajar por varios días, introducir bebidas alcohólicas para su consumo a las dependencias policiales, ya sea trabajando o fuera de servicio, presentarse al servicio en estado de embriaguez, conducir vehículos oficiales en estado de embriaguez, emborracharse mientras se cumple una sanción disciplinaria.

Si bien las bebidas alcohólicas cultural y socialmente hacen parte de casi todas las ceremonias y festividades de gran parte de la sociedad, en el caso de la institución policial es importante que esta reformule doctrinas, políticas y demás disposiciones que le permitan romper con este tipo de comportamiento consumista, para así impulsar en sus funcionarios la adopción de una forma de vida sin alcohol ni otras drogas. Esto bajo el entendimiento de que existe una relación de influencia bidireccional (institución a funcionarios y funcionarios a institución), ya que también el buen comportamiento de cada individuo influye positivamente para una mejor imagen de la Policía ante la población.

Se debe entender que el fenómeno comportamental no se origina exclusivamente en una sustancia, sino que presenta una fenomenología compleja, múltiple y sistemática de variada recursividad; que no podría ser entendida con una visión lineal o tradicional, sino que hace falta una teoría que conciba la complejidad. No se puede seguir permitiendo que los policías caigan en el consumo alcohol de forma tal que afecte su buen comportamiento dentro de las instancias administrativas y reglamentarias de la institución y del buen servicio que todo funcionario policial está obligado a brindar a la ciudadanía.

\section{Referencias}

Arnold, M. y Osorio, F. (1998). Introducción a los Conceptos Básicos de la Teoría General de Sistemas. Cinta de Moebio, (3). Recuperado de: http://www.redalyc.org/articulo.oa?id=10100306

De Puelles Casenave, C. (2008). Consumo perjudicial de alcohol en el cuerpo nacional de policía: implicaciones disciplinarias, laborales y psicopericiales. Psicopatología clínica, legal y forense, 8, 79-106. Recuperado de: http://www.masterforense.com/pdf/2008/2008art5.pdf 
Max-Neef, M. (2004). Fundamentos de la Transdisciplinaridad. Universidad Austral de Chile. Recuperado de http://ecosad.org/phocadownloadpap/otrospublicaciones/max-neef-fundamentos-transdisciplinaridad.pdf

Ministerio de Salud Pública del Ecuador (MSP) (2016). Atención integral del consumo nocivo de alcohol, tabaco y otras drogas. Protocolo Quito. Ecuador: Ministerio de Salud Pública. Dirección Nacional de Normatización-Comisión de Salud Mental-MSP. Recuperado de https://vlex.ec/vid/atencion-integralconsumo-nocivo-642159313

Ministerio del Interior del Ecuador (2016). Reglamento de Disciplina de la Policía Nacional del Ecuador. Ecuador: Policía Nacional del Ecuador. Recuperado de http://www.policiaecuador.gob.ec/wp-content/ uploads/downloads/2013/07/REGLAMENTO_DE_DISCIPLINA_DE_LA_POLICIA_NACIONAL. $\underline{\text { pdf }}$

Morin, E. (1994). La noción de sujeto. En D. Fried-Schnitman (Comp.), Nuevos paradigmas, cultura y subjetividad (pp. 67-89). Argentina: Editorial Paidós. Recuperado de http://ahau2012.blogspot.com/2006/04/ nocin-de-sujeto-en-morin.html

Morin, E. (1999). Los siete saberes necesarios para la educación del futuro (M. Vallejo, trad.), Paris: Unesco. Recuperado de http://unesdoc.unesco.org/images/0011/001177/117740so.pdf

Morin, E. (1999b). La cabeza bien puesta. Repensar la reforma Reformar el Pensamiento. Buenos Aires: Nueva Visión. Recuperado de: https://revistanotaalpie.files.wordpress.com/2014/05/la _cabeza bien_puesta_1979.pdf

Morin, E. (2011). Introducción al pensamiento complejo. Recuperado de: http://www.pensamientocomplejo.com.ar/docs/files/MorinEdgar_Introduccion-al-pensamiento-complejo_Parte1.pdf

Munné, F. (2003) ¿Qué es la complejidad? Barcelona: Universidad de Barcelona. Recuperado de https:// eleuterioprado.files.wordpress.com/2010/07/baixar-artigo-3.pdf

Organización Mundial de la Salud (OMS) (1992). CIE-10. Décima revisión de la clasificación internacional de las enfermedades. Trastornos mentales y del comportamiento. Descripción clínica y pautas para el diagnóstico. Madrid: Ed. Méditor.

Organización Panamericana de la Salud (OPS) y Organización Mundial de la Salud (OMS) (2015) Informe de situación regional sobre el alcohol y la salud en las Américas. Washington: OPS. Recuperado de http://www.paho.org/hq/index.php?option=com _ content\&view=article\&id=11108\%3A2015-regionalreport-alcohol-health\&catid=1893\%3Anews\&Itemid=41530\&lang $=$ es

Real Academia Española (RAE) (2009). Diccionario de la lengua española. Madrid: Espasa.

República del Ecuador (2008, octubre 20). Constitución de la República del Ecuador Decreto Legislativo. Registro Oficial 449. Ciudad Alfaro: Asamblea Constituyente Recuperado de http://ecuadorforestal.org/ wp-content/uploads/2010/05/CONSTITUCION_DE_LA_REPUBLICA_DEL_ECUADOR_20081.pdf

República del Ecuador Asamblea Nacional (2014). Código Orgánico Integral Penal (COIP). Quito: República del Ecuador. Recuperado de: http://www.epn.edu.ec/wp-content/uploads/2015/06/COIP1.pdf 